\title{
Emission lines in the optical spectrum of 3 Cen $A^{\star, \star \star}$
}

\author{
G. M. Wahlgren ${ }^{1}$ and S. Hubrig ${ }^{2}$ \\ ${ }^{1}$ Lund Observatory, University of Lund, Box 43, 22100 Lund, Sweden \\ 2 European Southern Observatory, Casilla 19001, Santiago 19, Chile \\ e-mail: shubrig@eso.org
}

Received 2 September 2003 / Accepted 10 February 2004

\begin{abstract}
Previously, weak emission lines had been detected at red wavelengths in the spectra of a limited sample of mid to late B type main sequence stars. A fuller description of the occurrence and origins of these lines has yet to be forwarded, in part due to the lack of observations detailing the spectral transitions involved. To address this deficiency, we present a line list of weak emission features found in the optical and near infrared spectral region of the chemically peculiar He-weak star 3 Cen A (HD 120709). Nearly 350 features, mostly associated with allowed transitions from high-excitation states of first ions, are catalogued along with identifications. Prominent among the emission lines are the spectra P II, Mn II, Fe II, Ni II and Cu II. Emission lines from Ca II, Si II and $\mathrm{Hg}$ II are also evident. Abundances are determined for several elements from synthetic spectrum fitting, with anomalies detected for the ions O I/II, P II/III and Si II/III. The LTE synthetic spectrum fitting also revealed that the low excitation $4 s-4 p$ transitions of Fe II predict an abundance that is greater than that determined from higher excitation $4 \mathrm{~d}-4 \mathrm{f}$ transitions. Several of these latter transitions have upper energy levels that are found to be associated with emission lines. We also present empirical considerations for the excitation processes leading to the weak emission lines.
\end{abstract}

Key words. line: identification - stars: abundances - stars: chemically peculiar - stars: emission - stars: individual: 3 Cen A

\section{Introduction}

It has recently been established that mid to late B type main sequence stars display weak emission lines (WEL) in the opticalred region of the spectrum. The nature of the emission is distinct from that displayed by the classical emission-line Be stars, which present strong emission from hydrogen Balmer lines, broadened as a result of rapid rotation. To the contrary, the WELs detected in the spectra of mid to late B type mainsequence stars arise from high-excitation states of single ions and have strengths that can be characterised by equivalent widths of typically up to a few tens of $m \AA$. These WELs present a full-width at half-maximum (FWHM) that is similar to that measured for the absorption lines, implying their formation at the same stellar rotation as the absorption lines. For slowly rotating stars their detection requires a reasonably high spectral resolution and high signal-to-noise $(S / N)$ ratio.

The first identifications of these WELs were made in the spectra of two chemically peculiar stars, the He-weak

Send offprint requests to: G. M. Wahlgren,

e-mail: glenn.wahlgren@astro.lu.se

* Based on observations obtained at the European Southern Observatory, La Silla, Chile, No. 65.L-0316 and Paranal, Chile No. 266.D-5655.

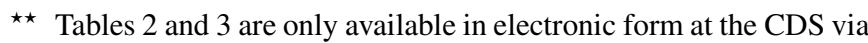
anonymous ftp to cdsarc.u-strasbg.fr $(130.79 .128 .5)$ or via http://cdsweb.u-strasbg.fr/cgi-bin/qcat?J/A+A/418/1073 star 3 Cen A (HD 120709, B5 III-IVp) and the HgMn star 46 Aql (HD 186122, B9 HgMn) (Sigut et al. 2000). From data obtained at high spectral resolution for two wavelength intervals, $\lambda \lambda 6105-6190,6350-6425 \AA$, WELs were identified from the spectra P II, Mn II, and $\mathrm{Hg}$ II for 3 Cen A, in addition to there being unidentified emission features. In the same wavelength regions only Mn II emission lines were observed in the spectrum of 46 Aql. The detection of weak emission lines was expanded by Wahlgren \& Hubrig (2000, 2001a) to a sample of 12 mid to late main sequence B stars of both normal and peculiar (HgMn) chemical composition. From two limited wavelength regions, $\lambda \lambda 6005-6095,6105-6190 \AA$, highresolution spectra allowed the detection of lines from Ti II, Cr II, and Mn II, and possibly Fe II. Correlations between elemental abundance and emission line strength were apparent. In the case of $\mathrm{Cr}$ II $\lambda 6182.3$ a moderately higher chromium abundance than the solar value is associated with stronger emission. Similarly, small abundance enhancements of manganese result in certain emission lines of Mn II, while a more extreme overabundance, as is evident in many $\mathrm{HgMn}$ stars, produces strong absorption lines.

A preliminary study for 3 Cen A (Wahlgren \& Hubrig 2001b), based upon the FEROS data used in the current work, announced the detection of WELs over a much larger wavelength region, $\lambda \lambda 5000-9200 \AA$, and attributed them to several first-ion species, including most elements of the iron group. One idea forwarded in that work was the possiblity for 
a temperature (spectral type) dependence of the emission features, in terms of the ability of ultraviolet continuum radiation to populate high-excitation states. According to Sigut (2001a,b), in normal B stars with $T_{\text {eff }}>13500 \mathrm{~K}$, Mn II multiplet 13 should appear in emission at the level of a few $\mathrm{m} \AA$, and the multiplet 13 emission line strength initially increases with manganese abundance but then reaches a maximum value and begins to diminish and the multiplet lines turn from emission to absorption. Temperature dependence was further studied through the observation of eight He-weak stars spanning the $T_{\text {eff }}$ range $17500 \mathrm{~K}$ to $13200 \mathrm{~K}$ (Wahlgren et al. 2003). The two hottest stars of the group, $3 \mathrm{Cen} \mathrm{A}\left(T_{\mathrm{eff}}=17500 \mathrm{~K}\right)$ and HD $185330\left(T_{\text {eff }}=16560 \mathrm{~K}\right)$, display more similar spectra in terms of emission line variety and strengths, while the cooler He-weaks stars may or may not show weak emission. A complication to this result is that the stars assigned to the $\mathrm{He}$ weak subclass P-Ga were likely to show emission lines while most other He-weak stars did not, thus admitting chemical peculiarity type to be a consideration.

Theoretical explanations for the WELs have been centered on interlocked non-LTE effects (Sigut 2001a,b), demonstrating that the presence of the emission from Mn II multiplet 13 can be qualitatively produced within the framework of a non-LTE treatment of the level populations coupled with a stratification of the manganese abundance. However, the relative strengths of multiplets are not accurately reproduced for $3 \mathrm{Cen} \mathrm{A}$, and further experimentation is needed to understand these discrepancies. For various multiplets of Mn II equivalent widths have been predicted based on simple stratified models and compare favorably with the limited observations. The possibility of selective excitation mechanisms has also been suggested (Wahlgren \& Hubrig 2000).

The purpose of the work presented here is not to derive a definitive model for the appearance of emission lines from different elements. It is our understanding that the advancement and testing of theoretical explanations for the origin of the emission lines would greatly benefit from the tabulation of observable emission lines. It is with this purpose that we have undertaken to catalogue the emission lines observed in the spectrum of 3 Cen A, a star for which the number of WELs and the variety of their attributed ions is the most extensive among the B stars studied to date.

\section{Observational data}

The data comprising this study cover essentially the entire optical wavelength region and extend out to $1 \mu \mathrm{m}$. Our original efforts (Wahlgren \& Hubrig 2001b) were based on spectra obtained with the echelle spectrograph FEROS (Fiber Extended Range Optical Spectrograph) on the $1.52 \mathrm{~m}$ telescope at La Silla. Later, it was learned that similar data had been obtained using the UVES (Ultraviolet Visual Echelle Spectrograph) on the $8 \mathrm{~m}$ VLT2 telescope at Paranal, and these data were incorporated into the analysis. Despite the fact that the UVES spectrum has a higher spectral resolution, the detection of WELs is not very sensitive to this parameter due to the widths of the features. A similarly good $S / N$ ratio for the two spectra allows for these two data sets to tell a more complete story about the WELs from the perspectives of completeness and potential for the detection of line variability.

The FEROS spectra were taken on the 16th and 17th of May 2000 with exposure times of 600 and 900 s, respectively. Each spectrum covers the wavelength region between 3530 and $9220 \AA$, and has a nominal resolving power of $R=48000$. The standard MIDAS pipeline for FEROS was used to define the echelle orders on flat-field frames, subtract the background, extract the echelle orders, apply flat field corrections to the extracted orders (including removal of pixel-to-pixel variations as well as correction for the blaze function), calibrate the wavelength scale and merge the 39 orders. The $S / N$ ratios of the resulting spectra are high, ranging between 100 to 200 in the one-dimensional spectrum, although the $S / N$ drops below 100 nearing the ends of some orders. Our measurements have been restricted to lines found within the free spectral range of each order, although we have used the features found within the regions of overlap for confirmation of emission.

Non-stellar spectral structures resembling emission features are present and are the result of noise spikes, cosmic rays, or data reduction artifacts. These spectral artifacts appear noticeably different for spectra taken on consecutive nights and the comparison of the two spectra provides a strong discriminant against misidentification of artifacts as stellar emission lines, assuming that the emission lines do not vary considerably from night to night.

The UVES spectra were recorded on the 24 June 2001 as part of the Paranal Observatory Project to create a library of high-resolution spectra across the Hertzsprung-Russell Diagram. Two exposures were made having a resolving power of $R=80000$. The flux levels are not identical but they scale nicely to present spectra of similar appearance with respect to $S / N$ characteristics and emission line strengths. The data retrieved from the UVES archives were reduced according to standard pipeline data processing procedures. The final reduced data set consists of six spectral segments that together span the wavelength interval $3100 \AA$ to beyond $1 \mu \mathrm{m}$ with the exception of gaps in the spectrum, the largest being between $8500-8650 \AA$, for which there is no data. The $S / N$ ratio is high $(>100)$ across most of the spectrum; however, at longer wavelengths $(\lambda>9000 \AA)$ the $S / N$ drops to values estimated to be as low as 50, which adversely affects the detection and measurement of WELs at these wavelengths. As for the FEROS data, the reality of an emission feature was tested by comparison of the feature in the two observations.

\section{Synthetic spectrum analysis}

As an aid to the process of identifying WELs, synthetic spectra were generated using the ATLAS/SYNTHE (Kurucz 1993) suite of programs. A model atmosphere was generated using the ATLAS9 code for the stellar parameters determined by Castelli et al. (1997) $\left(T_{\text {eff }}=17500 \mathrm{~K}, \log\right.$ gravity $\left.=3.8\right)$. This $T_{\text {eff }}$ was decided upon by Castelli et al. because it was deemed suitable to the study of $I U E$ spectra. Indications for $T_{\text {eff }}$ to be as high as $18000 \mathrm{~K}$, or perhaps higher, are evident from photometric and spectrophotometric methods. The initial elemental abundances in our analysis were taken from 
Table 1. Elemental abundances in 3 Cen A.

\begin{tabular}{lc}
\hline \hline Species & $\log N_{\mathrm{el}}$ \\
\hline C I & 7.17 \\
N I & 8.35 \\
N II & 8.50 \\
O I & 8.84 \\
O II & 8.14 \\
Al II & 5.43 \\
Si II & 7.25 \\
Si III & 8.09 \\
P III & 7.66 \\
Cr II & 5.48 \\
Mn II & 7.06 \\
Fe II & 7.80 \\
Fe III & 7.88 \\
Ba II & $\leq 3.1$ \\
Hg II & 4.55 \\
\hline
\end{tabular}

Adelman \& Pintado (2000) and Pintado et al. (1998), which had been derived from equivalent width analyses, and the copper abundance enhancement, $[\mathrm{Cu} \mathrm{II} / \mathrm{H}]=+2.0$, is that from Castelli et al. After we experimented with the turbulent velocity parameter, $v_{\mathrm{t}}$, a value of $0 \mathrm{~km} \mathrm{~s}^{-1}$ was chosen. The synthetic spectrum showed very small differences to changes in $v_{\mathrm{t}}$ between 0 and $1 \mathrm{~km} \mathrm{~s}^{-1}$, and we had no basis for choosing a nonzero value. The stellar absorption spectrum is sharp lined, and is well reproduced by synthetic spectra having a rotational velocity of $2 \mathrm{~km} \mathrm{~s}^{-1}$ for the instrumental resolution of UVES, as previously mentioned by Sigut et al. (2000).

Synthetic spectra were generated for the wavelength region 4000-4700 $\AA$ for the purpose of checking the published elemental abundances. Atomic line data were taken from the compilation of Kurucz (1995). The $g f$ values of Wiese et al. (1996) were used for lines of carbon, nitrogen and oxygen. Abundances were altered to match the observed data for spectral lines originating from lower excitation levels of the spectra P II, Mn II and Fe II, and available lines in the case of other elements $(\mathrm{C}, \mathrm{N}, \mathrm{O}, \mathrm{Si}, \mathrm{Al}, \mathrm{Cr}, \mathrm{Ba})$. Our fitting of the spectral lines lead to changes from the initial abundance values for a number of elements. Our revisions for certain elements, as determined from spectral lines of the identified species, are presented in Table 1 on a scale where $\log N_{\mathrm{H}}=12.00$. Figure 1 presents a sample of the synthetic spectrum fitting from the UVES data.

It became quickly evident that the goodness of the fits to the individual lines of an ion are of variable goodness and not likely to be the result of only poor $g f$ values. For the Fe II lines the fits were quite good using the final abundance value for lines having an upper energy level near $45000 \mathrm{~cm}^{-1}$. These $4 s-4 p$ transitions have experimentally determined $g f$ values. For lines involving upper energy levels of higher excitation $\left(E_{\text {upper }} \gtrsim 80000 \mathrm{~cm}^{-1}\right)$ the fit was less good and for transitions whose lower energy level was greater than approximately $90000 \mathrm{~cm}^{-1}$ the line would generally be observed in emission. There exist few, weak high-excitation lines of Fe II that are observed in absorption and are well fit by the synthetic spectra. Thus, the presence of spectral line fill-in (which we define here

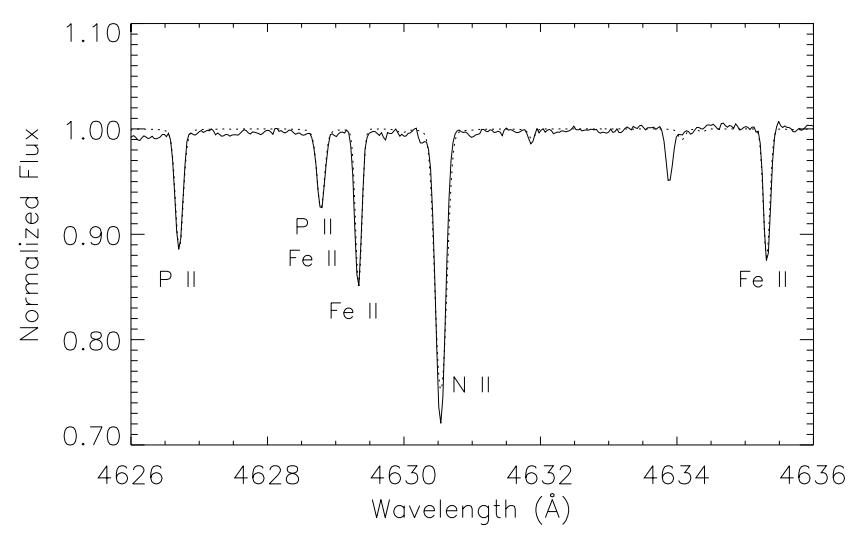

Fig. 1. A comparison of the UVES spectrum (solid) of 3 Cen A with the synthetic spectrum (dotted) shows good fits to low-excitation $(4 s-4 p)$ transitions of Fe II.

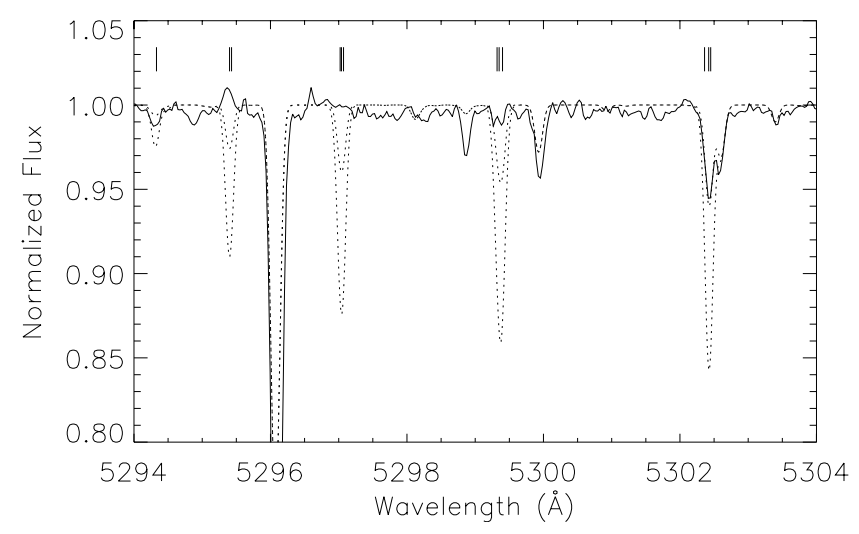

Fig. 2. The Mn II multiplet 11 in the FEROS spectrum of 3 Cen A. Two synthetic spectra are presented for comparison: $\log N_{\mathrm{Mn}}=-4.94$ (best fit abundance from Mn II lines) and -5.60 (best fit for the 5302.5 component). Emission above the continuum is evident for only the 5295.4 $\AA$ component, while the other components all display absorption line fill-in as measured from the best fit for the Mn II abundance. The locations of all multiplet lines are marked.

to be a weakened observed line profile relative to a synthetic profile that has been computed using an abundance that was determined from low-excitation transitions) or even presence of the weakest emission lines for the high-excitation states is a measure relative to results obtained from the low-excitation lines.

The manganese abundance is determined by fitting seven $4 s-4 p$ transitions of the Mn II spectrum found in the region $4242-4327 \AA$. Our value of $\log N_{\mathrm{Mn}}=7.06 \pm 0.1$ is similar to that determined by Adelman \& Pintado $\left(\log N_{\mathrm{Mn}}=7.20\right)$, despite their inclusion of three lines (Mn II $\lambda \lambda 5294,5299,5302$ ) that are found to be filled-in (see Fig. 2) and a scatter among individual line abundances that spans $1 \mathrm{dex}$. We attribute the large scatter for Mn II, and other ions, in that work to the use of equivalent widths and the mixture of lines from low and high excitation states, the latter reflecting the possible influence of weakened absorption lines from high-excitation states. 
Apparent ionization anomalies are present for the spectral pairs O I/II, P II/III and Si II/III and possible explanations would include improper atomic data, a violation of population of energy levels from Boltzman statistics (non-LTE) or perhaps the consequence of diffusion-induced vertical stratification of these elements in the stellar atmosphere. Also, the carbon abundance determined by us from optical lines of $\mathrm{C} \mathrm{I}$ is weaker than the abundance as determined using lines from IUE spectra (Castelli et al.). In the case of oxygen the O I triplet near $\lambda 7770 \AA$ is fit using an abundance that is essentially solar, while O II lines from multiplets 64, 72 and 99 (Wiese et al. multiplet numbering scheme) are well fit by a much lower abundance. These abundances, derived under the assumptions of LTE, are similar to those mentioned by Baschek et al. (1977), who discuss the abundance in terms of non-LTE corrections.

We found the abundance of phosphorous to be well represented on average by the value $\log N_{\mathrm{P}}=7.16$ from Adelman et al., but the fits to individual lines were rather erratic. We suggest that the P II spectrum be reanalysed for oscillator strengths, which may influence the magnitude of the difference in abundance as determined by the P II and P III spectra. Our phosphorous abundance from P III lines is considerably different from that of Pintado et al. $\left(\log N_{\mathrm{P}}=6.91\right)$ despite using the same lines and oscillator strengths.

An abundance for mercury is estimated from fitting a weak absorption line (equivalent width $=2.5 \mathrm{~m} \AA$ ) at $3984.00 \AA$ as $\mathrm{Hg}$ II $\lambda 3984$. The wavelength was determined by shifting the observation to match the synthetic spectrum at the experimentally measured wavelength of N II $3995.00 \AA$. The significance of the $\mathrm{Hg}$ II wavelength is that the feature can only be fit if the isotopic composition is comprised of mostly ${ }^{202} \mathrm{Hg}$ II $\lambda 3983.99 \AA$ with a contribution of no more than $20 \%$ from ${ }^{204} \mathrm{Hg}$ II $\lambda 3984.07 \AA$. Using $\log (g f)=-1.73$ from Dworetsky (1980) leads to an abundance of $\log N_{\mathrm{Hg}}=4.55$, on a scale where $\log N_{\mathrm{H}}=12.00$, when the feature is interpreted as only ${ }^{202} \mathrm{Hg}$. It is difficult to be more accurate than this given the influence of noise on this weak feature. A reasonable uncertainty of $\pm 0.01 \AA$ in alignment of the observed and synthetic spectra impacts the relative contributions of the isotopes and the mercury abundance. Our value of the mercury abundance is an order of magnitude smaller than that presented by Castelli et al. based on synthetic spectrum fitting of presumably the Hg II $\lambda 1942$ resonance line in IUE spectra. This discrepancy may not be significant in light of difficulties surrounding the treatment of line blending in $I U E$ spectra and the lack of considerations for isotopic and hyperfine line structure in that study.

The large wavelength coverage of the data allowed us to search for a number of other elements, in particular the lanthanides. No detections were made of the strongest lines from the doubly-ionized states of praseodymium or neodymium, two elements that often display line enhancements in chemically peculiar stars.

Finally, we cannot overstate the need for caution for LTE abundance analyses of B type stars regarding the use of lines originating from high-excitation states typically found at red wavelengths, since the use of such lines may produce a systematically lower abundance.

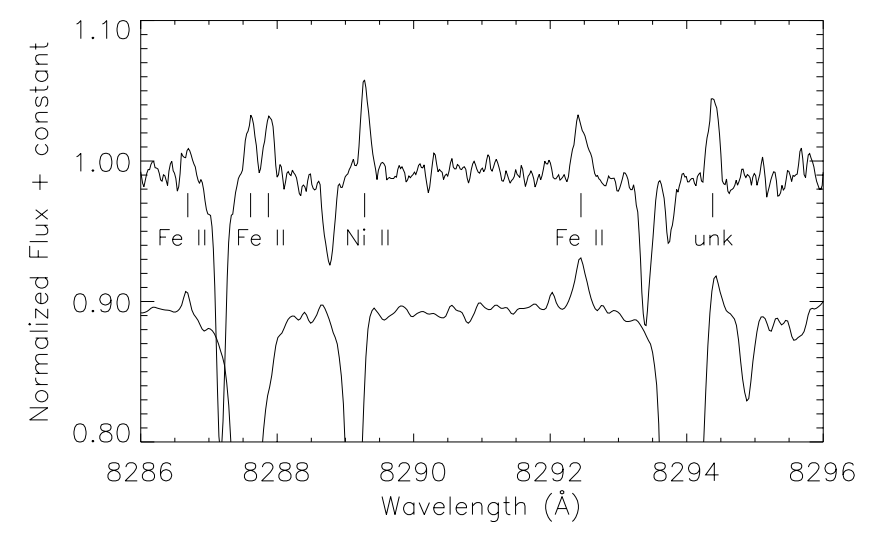

Fig. 3. The terrestrial absorption lines (all noteable absorption features) in the FEROS (lower) spectrum are deeper than in the UVES (upper) spectrum due to different conditions at the time of observation, and they are shifted between the two observations. Several emission lines become evident in the UVES spectrum as a result.

\section{The line list}

\subsection{Line list construction}

The WEL data are presented in two formats. Table 2 presents a listing of all features arranged in order of ascending wavelength. In Table 3 these same data, excluding lines of unknown (unk) or unsuspected identity, are grouped by multiplet for each ion and arranged in descending order of the term energy of the upper level of the transition. The primary criterion for cataloging a WEL candidate was its repeatability to be seen in emission in different spectra. For limited wavelength coverage, echelle order overlap for the FEROS instrument provided four spectra that could be reviewed for repeatability. For weak features, those at flux levels near that of the noise, the identifications were often supported by synthetic spectrum calculations that predicted the absorption line spectrum for the conditions of 3 Cen A. Therefore, the weakest features are more apt to be included as a result of their presence in the synthetic spectrum, especially if the line under consideration is a member of an ion or multiplet having other lines recognized to be in emission.

The UVES fluxed sky spectrum (Hanuschik 2003) was searched for coincidences with the emission lines listed in Table 2. For only the feature observed at $6300.02 \AA$ was there a reasonable coincidence with a sky-atlas line, in this case with an unidentified line at $6300.309 \AA$, the strongest feature in the atlas. The presence of an emission line at $6300 \AA$ peaks interest because of the possibility of it being [O III] $\lambda 6300.02$. This identification is very unlikely due to the absence of other lines from the same multiplet in the same FEROS spectrum. No emission feature was observed at this wavelength in the UVES spectrum.

The atmosphere of the earth is also influencial through its absorption line spectrum, which either can mask the stellar emission lines or affect the measurements of their wavelength and intensity. Fortunately, the FEROS and UVES spectra were taken at epochs that lead to a relative shift of approximately $0.4 \AA$ between their terrestrial spectra. This shift and its implications for the line list are illustrated in Fig. 3. 
The measurements of wavelength and equivalent width presented in the tables are primarily taken from the exposure on the first night for the FEROS data and the first observation of the UVES data, but are supplemented by data from the second spectrum of each when features in the primary spectrum appear to be influenced more highly by noise or the data reduction procedure. No credible evidence is apparent in the FEROS data that the emission features changed in their strength or wavelength from one night to the next. Notable differences in emission line equivalent width exist between the FEROS and UVES data for many lines. We attribute most of these differences to instrumental effects, noise, terrestrial absorption and the influence on the measurement of lines by the different spectral resolutions. However, there appear to be features for which the difference in measured equivalent width is unexplainable.

The observed wavelengths for WELs presented in the Tables 2 and 3 are representative of the emission feature. The wavelengths are those as measured in the UVES spectrum unless such data were unavailable. The lower spectral resolution of the FEROS data provides for a more uncertain wavelength. Synthetic spectra were computed for the entire wavelength coverage of each instrument and served as the laboratory or rest wavelength scale. For the FEROS data a wavelength shift was determined for each spectral order by comparison of the observed and computed absorption lines. This shift ranged in value from $-0.012 \AA$ to $-0.016 \AA$. In general, in the red spectral region there exist relatively few absorption lines in the spectra of B type stars. For most orders there existed a few suitable absorption lines, perhaps of different species. Although the lines are not evenly spaced in wavelength, their shifts were found to be fairly consistent across an order, to within the standard of error presented by the spectral noise characteristics. For a few orders no adequate absorption lines were available for determining a wavelength shift, and either a shift was determined from interpolating between orders or from emission line positions in regions of order overlap.

For the UVES spectra the wavelength shift was seen to vary with wavelength across the spectral segments constructed by concatenating the orders. These wavelength shifts were determined at the locations of each useful absorption line and do not necessarily vary in a monotonic manner across the entire spectral segment. The stellar absorption lines utilized came from the spectra N I, O I, Ne I, Mg II, P II and Fe II, with the Fe II lines prominent for $\lambda<6000 \AA$ and the Ne I lines prominent at longer wavelengths. The sparseness of stellar absorption lines along with the presence of regions dominated by terrestrial absorption lead to gaps in the spectrum (for example at 8780 to $9060 \AA$ ) where no useful stellar absorption lines exist for wavelength shift determination. The problem is most severe at the longest wavelengths, where no useful absorption lines were found longward of the O I 19266 line and the $S / N$ ratio is noticeably decreasing. Wavelength uncertainties here can be as high as $\pm 0.1 \AA$.

Where possible, two measures of wavelength were determined for each WEL, representing the peak of the emission and the center of the chord defining the full-width at half-maximum (FWHM). Due to the weakness of the emission features, the line profile was often influenced by the noise characteristics.
The two measures of wavelength were typically found to be in agreement to within $10 \mathrm{~m} \AA$, but were occasionally as large as $50 \mathrm{~m} \AA$. The wavelength based on the FWHM was found to be more reliable and it is this wavelength measure that was prioritized and typically presented in the tables. However, as a result of noise, line blending, or other peculiarities each spectral line was individually assessed and wavelengths based on the peak emission location are also used. In regions of high line density, in particular the regions of terrestrial absorption lines, the emission lines can be blended and their measured wavelengths shifted in accordance with the disturbing absorption line(s). The total uncertainty in the feature wavelength therefore is comprised of the intrinsic uncertainty ascribed to the individual absorption lines employed to set the wavelength rest frame, the spectrum noise and line blending. Intrinsic uncertainties can be as low as $1 \mathrm{~m} \AA$ for laboratory measured lines but are more typically of the order 5-10 m $\AA$. For the purposes of line identification, uncertainties of up to $50 \mathrm{~m} \AA$ can be meaningful. However, the implication to determining velocity shifts from individual lines is damaging and a high signal-tonoise spectrum should be procured to study velocity systematics using a higher spectral resolving power than provided by the UVES. For wavelengths longward $8000 \AA$ we note a suspicious shift of Ni II lines with respect to Fe II. Most lines of Ni II are observed to be blueshifted by 10 to $30 \mathrm{~m} \AA$ from their catalogued wavelengths, while Fe II lines in the same wavelength region are found to lie within $10 \mathrm{~m} \AA$ of their catalogued values. The difference may be the result of uncertainties in the $\mathrm{Ni}$ II and Fe II energy levels.

The intensities of the WELs are characterized by the equivalent width above a continuum level set with the aid of the synthetic spectrum. The spectral orders were not normalized prior to measurement, as the process of normalization makes assumptions about the continuum level that can mask the identification of the weakest emission features. The equivalent widths were measured using an Interactive Data Language (IDL) procedure. Depending upon the difficulty in defining the local continuum level, up to three measurements were made for a spectral line, defined by the most likely continuum level and reasonable estimates of its bounds. The equivalent width measurements were averaged and are presented to single decimal point accuracy in the tables. The uncertainties in the equivalent widths depend upon the continuum noise level and the strength of the observed emission. The stronger emission features $\left(W_{\lambda}>\right.$ $10 \mathrm{~m} \AA$ ) have uncertainties on the order of $10-30 \%$, while weaker features typically have uncertainties of $50 \%$. Lines for which $W_{\lambda}<1 \mathrm{~m} \AA$ should be considered to have a still higher uncertainty.

\subsection{Line list description}

In both Tables 2 and 3, the observed air wavelength, $\lambda_{\text {obs }}$, representative of the WEL and its equivalent width, $W_{\lambda}$, above the continuum are presented in Cols. 1 and 2, respectively. Wavelengths are presented from the UVES data set, and from the FEROS spectra in the absence of such UVES data. Equivalent widths are provided from both data sets in the 
ordering FEROS/UVES as positive numbers in the tables, despite the convention of using a negative value for emission lines and a positive value for absorption lines. The singular exception is made for the lines Mn II $\lambda 5294.3$ and $\lambda 5302.4$, for which the quoted values are for absorption features and are included to for completeness of presentation for multiplet 11 . Notable discrepancies between observed and computed absorption line depths, which we attribute to stellar atmospheric origins are acknowledged through suspected absorption line fill-in using the notations of acl for total fill-in of the computed spectral line at the continuum level and by $f i$ to denote suspected fill-in with a remaining absorption line component present. This interpretation is naturally influenced by the elemental abundances and line oscillator strength data, the latter being based solely upon theoretical formalisms for high excitation states such as those attributed to the emission lines. In recognition of these problems, we have been conservative in the attribution to line fill-in. Spectral lines suspected of being filled-in have only been included in the line lists if they share the same upper level as another line that shows emission and the observed and computed spectra were particularly discordant. This criterion accounts for only a small number of the Fe II lines that are computed to be too strong relative to the observation. For some features the synthetic spectrum provided convincing evidence that an emission feature was present, although it may be difficult to provide a meaningful measurement of wavelength and/or $W_{\lambda}$, perhaps due to noise or line blending. For these cases only the transition of the suspected emission feature is provided.

For each observed feature we have attempted to identify the responsible spectral transition(s). These data (air wavelength $\lambda_{\text {id }}$, ionic species, $\log g f$, the energy of the lower and upper levels of the transition, and the combination of configurations comprising the multiplet) are all extracted from the line list data of Kurucz (1995), with the exception of all data for P II (Svendenius et al. 1983), wavelengths for the Mn II $\left({ }^{6} \mathrm{~S}\right) 4 \mathrm{~d}$ e ${ }^{5} \mathrm{D}-\left({ }^{6} \mathrm{~S}\right) 4 \mathrm{f}{ }^{5} \mathrm{~F}$ multiplet lines (Johansson et al. 1995), and several energy level assignments for Fe II (Johansson, private communication). The last column in both tables provides the absorption line depth (Depth) computed by the SYNTHE program for the line center, on a scale where the normalized continuum level has a value of 1.000 . Thus, the observed equivalent width can be correlated against log $g f$ and a depth indicator that also accounts for the transition energy levels. In many cases Table 2 provides multiple potential contributors to a feature, whereas in Table 3 each of these contributors is assigned the full value of $W_{\lambda}$. Caution must therefore be exercised in interpreting the tabular data.

\section{Comments on emission lines}

The weak emission lines of Mn II have been receiving attention from both observational and theoretical perspectives. The lines of Mn II multiplet 13 (Fig. 4) have been observed in emission for stars from mid to late B spectral type and for normal and peculiar chemical composition. Therefore, their excitation mechanism must be common to this range of physical parameters in these stellar atmospheres. In the spectrum of $3 \mathrm{Cen} A$ the Mn II multiplet 11 lines (Fig. 2) also display evidence of

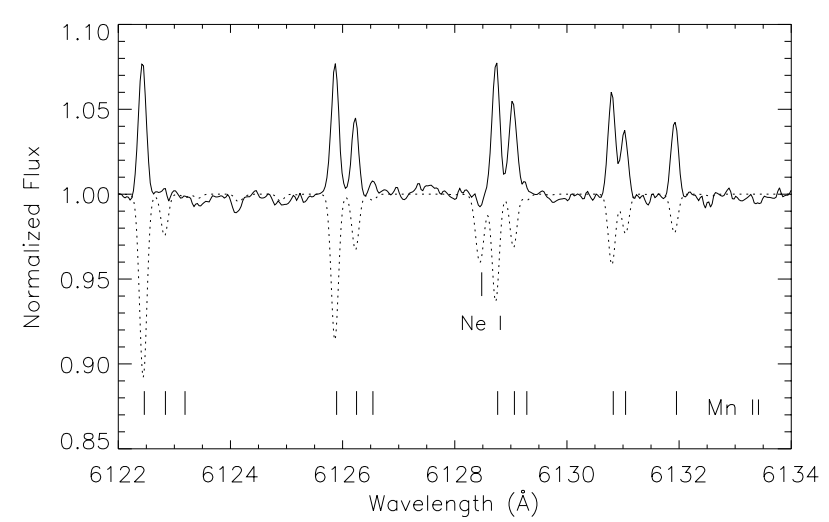

Fig. 4. The Mn II multiplet 13 in the FEROS spectrum (solid line) of 3 Cen A. The emission line strengths for Mn II multiplets 11 and 13 do not follow the relative proportions of the $g f$ values, as is discernible from the synthetic absorption line spectrum (dotted).

emission. Both multiplets have similarities in the values for their upper and lower energy levels, and the $g f$ values are also quite similar. For both multiplets the appearance of the emission or fill-in does not reflect the relative line strengths dictated by the oscillator strengths, as can be discerned from the synthetic spectrum calculations. This behavior has also been pointed out for the lines of Mn II multiplet 13 in other B stars (Wahlgren \& Hubrig 2000, 2001a).

In addition to these two multiplets, emission lines have been detected in the spectrum of 3 Cen A from higherexcitation Mn II multiplets. Emission from two of the highest energy multiplets in Table 3, corresponding to the emission lines at 8215.68 and $9128.61 \AA$, should be viewed with a little skepticism since there exists unpublished evidence (Johansson, private communication) for unidentified Fe II lines to be near these wavelengths. Unfortunately, certain multiplets are particularly plagued by terrestrial absorption, as for example $\left({ }^{4} \mathrm{G}\right) 5 \mathrm{~s} \mathrm{e}{ }^{5} \mathrm{G}-\left({ }^{4} \mathrm{G}\right) 5 \mathrm{p} \mathrm{w}{ }^{5} \mathrm{G}$, where only a single line has been detected and even it is affected by terrestrial absorption. A similar situation exists for the multiplet $\left({ }^{4} \mathrm{G}\right) 5 \mathrm{~s} \mathrm{e}{ }^{5} \mathrm{G}-\left({ }^{4} \mathrm{G}\right) 5 \mathrm{p} \mathrm{s}{ }^{5} \mathrm{~F}$, for which only the $\lambda 8215.688$ line has been detected for the same reason. The emission from the highest observed excitation, involving the transition $\left({ }^{6} \mathrm{~S}\right) 4 \mathrm{f}{ }^{7} \mathrm{~F}-\left({ }^{6} \mathrm{~S}\right) 6 \mathrm{~g}{ }^{7} \mathrm{G}$, is a complex feature that extends over $0.4 \AA$ and includes an absorption line of Fe II that may also be filled-in. This feature is observed to be in absorption in the FEROS spectra but its emission nature is noticed at the higher resolution of the UVES. We have presented an equivalent width for this feature as a lower limit, believing that in the absence of the Fe II absorption line the equivalent width would be much higher.

Emission is observed from the population of the ${ }^{6} \mathrm{~S}$ parent term configurations $\left(\geq 108000 \mathrm{~cm}^{-1}\right)$, which might substantiate the notion of radiative capture. Such levels produce lines in the region of our spectra having oscillator strengths that are one to two orders of magnitude weaker than the multiplet 11 and 13 lines, thereby making their detection more difficult. Emission can be traced along a decay chain defined by the terms $\left({ }^{6} \mathrm{~S}\right) 5 \mathrm{~g}{ }^{5} \mathrm{G}\left(E=108556.10 \mathrm{~cm}^{-1}\right) \rightarrow$ $\left({ }^{6} \mathrm{~S}\right) 4 \mathrm{f} \quad{ }^{5} \mathrm{~F} \quad(98464.20) \rightarrow\left({ }^{6} \mathrm{~S}\right) 4 \mathrm{~d} \quad$ e ${ }^{5} \mathrm{D} \quad(82144.48) \rightarrow$ $\left({ }^{4} \mathrm{G}\right) 4 \mathrm{p} \mathrm{z}{ }^{5} \mathrm{~F}$ (66 901.44). 


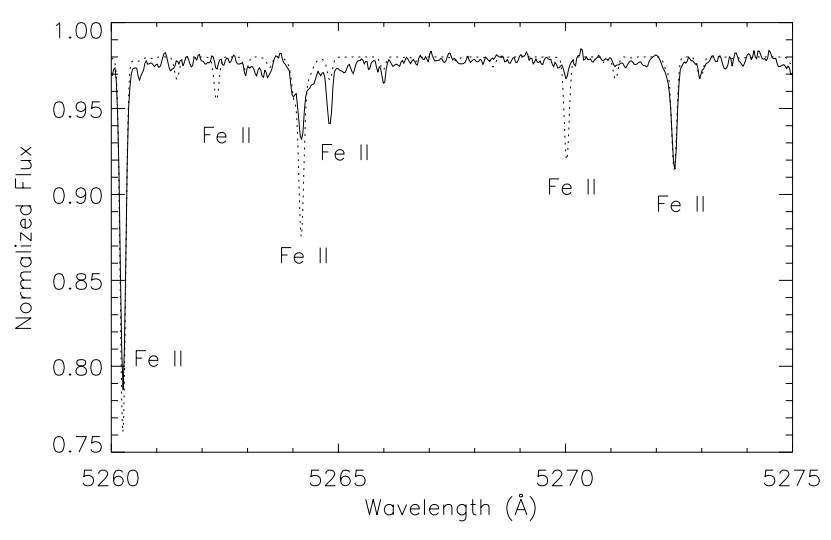

Fig. 5. Poor spectrum fitting of Fe II lines is common for lines for the $4 \mathrm{~d}-4 \mathrm{f}$ transitions. A single low excitation $4 \mathrm{~s}-4 \mathrm{p}$ transition at $\lambda 5272.25$ is present in this figure using the FEROS spectrum, and is well fitted by the abundance $\log N_{\mathrm{Fe}}=+7.80$.

The majority of WEL identifications are associated with Fe II transitions that originate from upper levels that range from $114104 \mathrm{~cm}^{-1}$ down to $61512 \mathrm{~cm}^{-1}$ above the ground state. As with Mn II, the path of Fe II photons can in some cases be traced through several stages of decay, up to three when arranged according to energy (for example, $104588.71 \rightarrow$ $89331.20 \rightarrow 78525.41 \rightarrow 62171.62 \mathrm{~cm}^{-1}$ ), along with many trains of three decay states when arranged by term structure. It seems apparent that radiative cascades are occurring, although their origin is unclear. Cascading may result after electron capture or the selective population of highly excited levels. As an alternative to cascades, emission of Mg II in B type stars has been shown to result from interlocked lines in non-LTE modeling using classical photospheric models (Sigut \& Lester 2000).

As mentioned earlier, the appearance of filling-in of absorption lines is suspected from synthetic spectrum fitting. In particular, many of the $4 \mathrm{~d}-4 \mathrm{f}$ transitions could be considered as filled-in ( $f i$ or acl designation) but they were not included in Tables 2 or 3 since they could not be directly connected to a level associated with observed emission (Fig. 5). Figure 6 presents an example of a filled-in Fe II line that does share its upper level with a line observed to be in emission.

The appearance of filled-in absorption lines could also result from errant $g f$ values. All of the $g f$ values for lines associated with high-excitation states are theoretical values, but the $4 \mathrm{~d}-4 \mathrm{f}$ transitions are not regarded as being especially troublesome for calculations. Observations of the cooler $\left(T_{\mathrm{eff}}=\right.$ $10750 \mathrm{~K}$ ), HgMn star HR 7775 (Johansson et al., in preparation) show the $4 \mathrm{~d}-4 \mathrm{f}$ transitions to be as well matched as the lower excitation Fe II lines for the same iron abundance. Therefore, the $g f$ values are not likely to be incorrect by significant amounts and are not the cause of the weakened absorption lines. This is significant for two reasons. First, atomic theorists looking for guidance from astronomical spectra should be cautious as to whether observed line profiles of high-excitation lines are influenced by selective excitation, radiative cascades and deviations from the Boltzman distribution for the population of levels. Secondly, elemental abundances determined in LTE from high-excitation lines can provide a systematic error of several tenths of a dex compared with an abundance

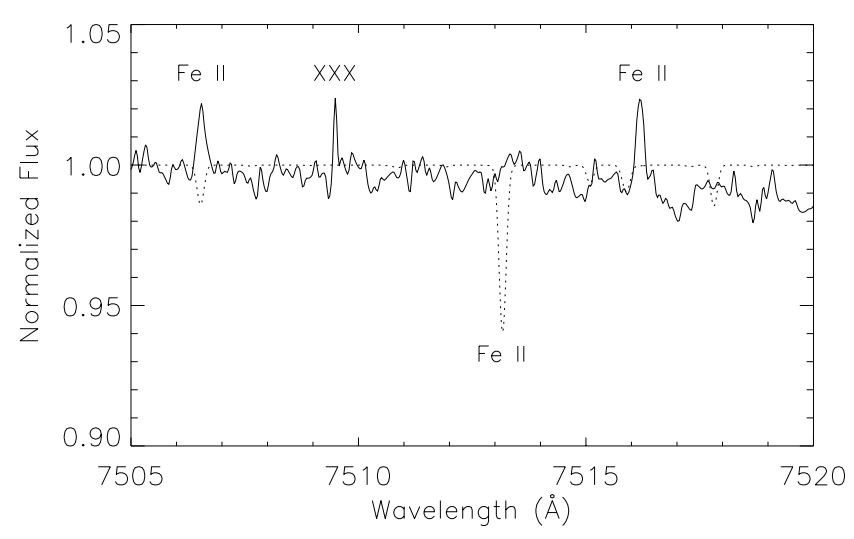

Fig. 6. Fill-in of absorption lines is revealed by the synthetic spectrum fitting. The transition Fe II $\lambda 7513.162$ shares its upper energy level with the line $\mathrm{Fe}$ II $\lambda 7731.673$, the latter being observed in emission in the 3 Cen A spectrum. Also present in this figure utilizing FEROS data are emission lines of Fe II and a spectral artifact designated by the symbol "xxx".

determined from low-excitation lines. At red wavelengths the line blending is less severe in B type stars than at blue wavelengths, where lines from lower excitation states are found, thereby making the red region of the optical spectrum an enticing one for abundance analyses. However, it is at redder wavelengths $(\lambda>5000 \AA)$ where many high-excitation transitions are found, and which can be afflicted by problems with energy level assignment and calculated oscillator strengths.

The assignment of a WEL observed at $6149.50 \AA$ to the spectrum of $\mathrm{Hg}$ II by Sigut et al. (2000) would benefit from confirmation by additional lines of $\mathrm{Hg}$ II viewed in emission. To this end we have searched for other lines of $\mathrm{Hg}$ II and detected a strong emission feature at a wavelength coinciding with the other line of the $7 \mathrm{~s}^{2} \mathrm{~S}-7 \mathrm{p}{ }^{2} \mathrm{P}$ multiplet, at $7944.414 \AA$. However, this emission feature is likely to have a strong contribution from the line $\mathrm{Cu}$ II $\lambda 7944.438$, if the assumption is made that the equivalent widths for the $\mathrm{Cu}$ II emission lines should be in proportion to their $g f$ values in the multiplet. Investigation of other optical $\mathrm{Hg}$ II lines provided no additional insights. Several lines of $\mathrm{Hg}$ II $(\lambda \lambda 4855.6,5425.6,5677.3)$ were undetected. The latter two lines possess highly uncertain or unknown $g f$ values, thereby making it impossible to reliably determine the extent of absorption line fill-in or consistency among the different lines. The upper level of the $\lambda 4855$ line also serves as the lower level of the $\lambda 6149$ line, for which emission is suspected. However, there is neither emission nor absorption for the $\lambda 4855$ line. An absorption feature interpreted as $\mathrm{Hg}$ II $\lambda 3984$ was earlier discussed for its use in estimating the abundance of mercury.

\section{Discussion}

We present a listing of weak emission lines detected in the optical spectrum of the He-weak star 3 Cen A. The spectra P II, $\mathrm{Mn}$ II, Fe II, Ni II and Cu II are particularly rich in the number of emission lines. In addition, the presence of emission lines from $\mathrm{Si}$ II, Ca II, and $\mathrm{Hg}$ II appears convincing, despite the small number of lines observed. The reality of emission from 
the spectra C I, N I, N II, Cr II and Ti II should be considered highly suspect since the few lines involved do not appear to have a mechanism for excitation that would not involve other lines of the species, and most of the observed features have an additional identification attached to them. Of the 74 features designated as unknown, $45(60 \%)$ may originate from the Fe II spectrum (Johansson, private communication), although they are still of undetermined energy levels. For wavelengths longward of $8000 \AA$ this percentage increases to $70 \%$ (38 of 55 lines). These same unpublished data suggest that other WELs may have strong contributions, if not in their entirety, from the Fe II spectrum. Of particular note in this regard are the lines Mn II $\lambda \lambda 8215,9128$ that arise from among the highest excitation states listed for this spectrum in the tables. Another indication that these Mn II lines may not be responsible for the observed features is the lack of any evidence for emission from lines having stronger $g f$ values in the multiplets involved.

The need to account for element stratification in 3 Cen A is underlined by the likely non-terrestrial isotopic composition of mercury, as noted through the Hg II $\lambda 3984$ feature. Models including stratification also point to this as being significant. Table 2 of Sigut (2001a) compares observed equivalent widths with those predicted for specific cases of uniform and stratified manganese distributions. From the UVES spectra used in this study the equivalent widths of several of these lines have been presented in our tables. For three Mn II lines not presented in our tables we note that their equivalent widths are $6.7,8.1$, and $3.6 \mathrm{~m} \AA$, respectively, for the lines at 4326.63, 4755.73, and $4806.82 \AA$. For these three weak lines their equivalent widths would appear to be better represented by the stratified model. We note, however, that for the case of uniform manganese abundance the predicted equivalent widths for these three lines are essentially half that of the observed values, a difference of 0.3 dex in the abundance.

Based on the data presented in the tables we put forth considerations for theories aimed at explaining the nature of the excitation process(es) leading to the formation of the emission lines in 3 Cen A. As multiple processes may be required to account for all of the lines observed, we look for insights that go beyond the predictions of classical non-LTE modeling. In evaluating the plausibility of any particular mechanism it is therefore beneficial to question why certain ions present emission while others do not. The solutions will need to include considerations of the atomic structure, elemental abundances, and the radiation field. A close binary companion has yet to be detected for 3 Cen A; however, the plausibility of a significant ultraviolet radiation source, perhaps in the form of a white dwarf, should not be ignored.

The presence of emission lines could imply that there exists a region of the upper atmosphere where the number density of an ion is low enough for the occurrence of radiative decays to produce allowed transitions, yet not forbidden transitions. This atmospheric region shares the same stellar rotation as the absorption line spectrum, as dictated by current observations that the emission lines do not appear to be shifted in wavelength from the position of the underlying absorption line, and their FHWM are similar to those of absorption lines. The location and extent of this region has not been established, despite certain qualitative agreement in producing emission lines with classicial one-dimensional photospheric models and non-LTE physics. These simple model atmospheres neglect the influences of magnetic fields, stellar winds, and diffusion, that are active in the atmospheres of B type stars and which may significantly alter the atmospheric structure predicted by onedimensional LTE models.

In this low density regime collisional excitation can be responsible for populating metastable states, such as exist for the iron-group elements. For kinetic temperatures in the range $10000-20000 \mathrm{~K}$ energy levels lying below 7000 to $14000 \mathrm{~cm}^{-1}$ above the ground state can be reached. This energy range is sufficient to include such lower metastable states as Mn II $3 d^{5}\left({ }^{6} \mathrm{~S}\right) 4 \mathrm{~s}$ a ${ }^{5} \mathrm{~S}\left(9472.97 \mathrm{~cm}^{-1}\right)$ and $3 \mathrm{~d}^{6}$ a ${ }^{5} \mathrm{D}$ near $14500 \mathrm{~cm}^{-1}$. For the Fe II spectrum the terms of interest would include $3 \mathrm{~d}^{6}\left({ }^{5} \mathrm{D}\right) 4 \mathrm{~s}$ a ${ }^{4} \mathrm{D}\left(7955-8846 \mathrm{~cm}^{-1}\right)$ and $3 \mathrm{~d}^{7}$ a ${ }^{4} \mathrm{P}$ (13474-13904 $\mathrm{cm}^{-1}$ ). Pumping of the metastable levels by continuum radiation at ultraviolet wavelengths in the region 1000-1300 $\AA$ can then excite ions up to levels at high energies. Ions in these high-energy states would then immediately cascade downward through lower energy levels, emitting radiation for allowed transitions along the decay path, which we detect via emission lines at optical-red wavelengths.

Several observations are of relevance to this picture. The first is that both 3 Cen A and HD 185330, the hottest stars studied to date that display WELs, show emission of ions and lines that are not observed in the spectra of cooler B stars. Therefore, a temperature dependence of the emission species could reflect the higher intensity of continuum radiation below H Ly $\alpha$ that would be available to pump ions from metastable states. In addition, the hotter nature of these two stars allows for metastable states of higher energy to be populated. For example, B stars covering the entire range of $T_{\text {eff }}$ from 17500 down to $11000 \mathrm{~K}$ have been observed to show emission of the Mn II multiplet 13. The upper levels of these lines can be populated by absorption of ultraviolet radiation in the vicinity of $1200 \AA$ by metastable states found at $9400 \mathrm{~cm}^{-1}$. However, for any of the higher excitation multiplets of Mn II to be excited in this manner the radiation would have to come from wavelengths as low as $1000 \AA$, for which the coolest B stars are deficient.

Observed emission lines from the Fe II spectrum originate from levels as high as $114000 \mathrm{~cm}^{-1}$, and have direct branches to metastable levels. As an example, the strong observed emission at $\lambda 9112.025$ has its upper level $\left({ }^{3} \mathrm{H}\right) 5 \mathrm{p}{ }^{2} \mathrm{G}_{4.5}$ at $110064.95 \mathrm{~cm}^{-1}$. This level connects to the level $\left({ }^{3} \mathrm{~F}\right) 4 \mathrm{~s} \mathrm{a}{ }^{2} \mathrm{~F}_{3.5}$ at $27314.922 \mathrm{~cm}^{-1}$ via radiation at $\lambda 1208.559 \AA$, a wavelength that has been claimed to show an emission feature in the spectra of the hot-A stars Sirius and Vega (van Noort et al. 1998). From that work, nine lines of Fe II and three lines of Cr II that are claimed to produce emission at ultraviolet wavelengths have upper levels that are producing emission lines in the 3 Cen A spectrum.

A second observation of note is that the emission lines for 3 Cen A are dominated by iron-group elements and phosphorus, which all have metastable levels that can be excited to highexcitation states through ultraviolet radiation (1000-1300 ̊). The immediate post iron-group elements $\mathrm{Zn}, \mathrm{Ga}$, and Ge do not have metastable levels and we have not associated any emission 
lines with these elements. The situation for gallium is of particular interest because of its strong abundance enhancement $([\mathrm{Ga} / \mathrm{H}]=+3.25 \mathrm{dex}$, Castelli et al. 1997) in 3 Cen A and other chemically peculiar stars of spectral type B.

Another important consideration for the production of emission lines is electron capture by a doubly-ionized atom. The $T_{\text {eff }}$ of 3 Cen A is sufficiently high that the doubly-ionized stage would be the dominant one for many elements. Strong lines from doubly-ionized species, including P III, Mn III, Fe III, Ni III, and $\mathrm{Cu}$ III, are present in the IUE spectrum of 3 Cen A. Emission lines originating from the highest excitations for Mn II, Fe II, and Ni II have parent terms representing the ground term of the doubly-ionized state and all metastable states that extend up to an excitation energy just under $30000 \mathrm{~cm}^{-1}$. For Si III and P III there are no metastable states and no excited levels below $50000 \mathrm{~cm}^{-1}$, so all recombinations occur from the ground state.

The data for the WELs listed in Tables 2 and 3 should be of sufficient number and quality to forward investigation into the nature of emission lines in $3 \mathrm{Cen} \mathrm{A}$ and the phenomenon of weak emission lines in the spectra of main sequence B stars. Spectra of even higher quality than those presented here would no doubt both increase the number of detected WELs as well as provide a higher accuracy for their equivalent widths and observed wavelengths. Further detection of the weakest features would help to fill gaps in our knowledge about the energy levels involved in the emission. Improvements in the accuracies of the observed wavelengths and equivalent widths are required to detect systematic velocity shifts of $\leq 1 \mathrm{~km} \mathrm{~s}^{-1}$ that may exist among different species and between the emission and absorption line systems.

Acknowledgements. We would like to thank S. Johansson for the use of unpublished data for the Fe II spectrum and discussions pertaining to excitation processes.

\section{References}

Adelman, S. J., \& Pintado, O. I. 2000, A\&A, 354, 899

Baschek, B., Scholz, M., \& Sedmayr, E. 1977, A\&A, 55, 375

Carlsson, M., Rutten, R. J., \& Shchukina, N. G. 1992, A\&A, 253, 567

Castelli, F., Parthasarathy, M., \& Hack, M. 1997, A\&A, 321, 254

Dworetsky, M. M. 1980, A\&A, 84, 350

Hanuschik, R. W. 2003, A\&A, 407, 1157

Johansson, S., Wallerstein, G., Gilroy, K. K., \& Joueizadeh, A. 1995, A\&A, 300, 521

Kurucz, R. L. 1993, Kurucz CD-ROM 18

Kurucz, R. L. 1995, Kurucz CD-ROM 23

Migdalek, J. 1976, Can. J. Phys., 54, 2272

Pintado, O. I., Adelman, S. J., \& Gulliver, A. F. 1998, A\&AS, 129, 563

Sigut, T. A. A., \& Lester, J. B. 1996, ApJ, 461, 972

Sigut, T. A. A., Landstreet, J. D., \& Shorlin, S. L. S. 2000, ApJ, 530, L89

Sigut, T. A. A. 2001a, ApJ, 546, L115

Sigut, T. A. A. 2001b, A\&A, 377, L27

Svendenius, N., Magnusson, C. E., \& Zetterberg, P. O. 1983, Phys. Scr., 27, 339

van Noort, M., Lanz, T., Lamers, H. J. G. L. M., et al. 1998, A\&A, 334,633

Wahlgren, G. M., \& Hubrig, S. 2000, A\&A, 362, L13

Wahlgren, G. M., \& Hubrig, S. 2001a, in Magnetic Fields Across the Hertzsprung-Russell Diagram, ed. G. Mathys, S. K. Solanki, \& D. T. Wickramasinghe (San Francisco: ASP), ASP Conf. Ser., 248, 369

Wahlgren, G. M., \& Hubrig, S. 2001b, in ASP Conf. Ser., 248, 365

Wahlgren, G. M., Hubrig, S., \& Ivarsson, S. 2003, in proceedings from the International Conference on Magnetic Fields in O, B, and A Stars: Origin and Connection to Pulsation, Rotation and Mass Loss, ed. L. A. Balona, H. F. Henrichs, \& R. Medupe (San Francisco: ASP), ASP Conf. Ser., 305, 256

Wiese, W. L., Fuhr, J. R., \& Deters, T. M. 1996, Atomic Transition Probabilities of Carbon, Nitrogen, and Oxygen, Journ. Phys. Chem. Ref. Data, Monograph 7 (NIST: Gaithersburg, MD) 